\title{
Psicología Jurídica en España: Delimitación Conceptual, Campos de Investigación e Intervención y Propuesta Formativa dentro de la Enseñanza Oficial
}

\section{Legal Psychology in Spain: Conceptual Boundary, Fields of Investigation and Intervention and Formative Proposal of Official Education}

\author{
José Manuel Muñoz \\ Antonio L. Manzanero \\ Miguel Ángel Alcázar \\ Comunidad de Madrid, España \\ Universidad Complutense de Madrid, España Universidad Autónoma de Madrid, España \\ José L. González \\ $M^{a}$ Luisa Pérez \\ María Yela \\ Policía Judicial de la Guardia Civil, España \\ Comunidad de Madrid, España \\ Instituciones Penitenciarias, España
}

Resumen. La Psicología Jurídica como especialidad profesional del psicólogo ha tenido un desarrollo exponencial desde finales de los años ochenta hasta la actualidad, considerándose un campo consolidado dentro de la Psicología Aplicada.

Inexplicablemente, sin embargo, esta madurez de la Psicología Jurídica no se ha visto reflejada con su incursión en los nuevos itinerarios curriculares elaborados a partir del Espacio Europeo de Educación Superior.

En el presente trabajo se maneja como hipótesis explicativa de esta situación la dificultad de vertebrar este campo disciplinar debido a los diversos ámbitos de interés investigador y de intervención.

Se pretende, por tanto, generar debate entre académicos y profesionales de la Psicología Jurídica con el fin de buscar el consenso que impulse un firme afianzamiento no sólo de perfiles profesionales específicos dentro del ámbito de la Psicología Jurídica sino de ésta como disciplina dentro de la Psicología.

Se propone así, un concepto único para definir el campo, distintas áreas en las que agrupar la investigación y prácticas profesionales, a la vez que se sugiere un proyecto de Master Oficial en Psicología Jurídica como punto de partida para el debate.

El reconocimiento académico de la Psicología Jurídica además de garantizar la proyección futura de la disciplina tanto en su vertiente aplicada como de investigación permitirá seguir ofreciendo a la sociedad en general y al mundo de la ley en particular una actuación profesional de calidad y adaptada a los retos futuros.

Palabras clave: Espacio Europeo de Educación Superior, Master, Psicología Jurídica.

Abstract. Legal Psychology as psychologist's professional specialty has had an exponential development since the end of the Eighties until the present time, considering itself a consolidated field within Applied Psychology.

Inexplicably, nevertheless, this maturity of Legal Psychology has not been reflected with its incursion in the new curricular itineraries elaborated from the European Space of Superior Education.

La correspondencia sobre este artículo dirigirla al primer autor al email: jmvforensic@yahoo.es 
This paper handles, as an explanatory hypothesis of this situation, the difficulty of integrating this disciplinary field due to the diverse scopes of investigative interest and intervention. It is intended, therefore, to generate debate between academics and professionals of Legal Psychology in altars to the search of the consensus that impels a firm reinforcement not only of specific professional profiles within the scope of Legal Psychology but also as a discipline within Psychology.

A unique concept to define the field, different areas in which to group the professional investigation and practices, are being proposed, as well as it is being suggested a project of Official Masters in Legal Psychology as departure point for the debate.

The academic recognition of Legal Psychology, along with guaranteeing the future projection of this discipline in its applied and investigative areas, will allow to continue offering a professional and qualified performance adapted to future challenges, to the mainly society and specifically the world of the law.

Keywords: European Higher Education Area, Legal Psychology, Master.

\section{Introducción}

Atendiendo al interés del Derecho, la regulación de la convivencia social, es clara la aportación que la Psicología puede realizar como ciencia que explica la conducta. De ahí que la colaboración entre ambas cuente con una larga historia que surge con el nacimiento del Derecho y se ha desarrollado con el surgimiento de la Psicología científica desde finales del siglo XIX (Manzanero 2010; Carpintero 2006; Sáiz y Sáiz, 2006). En España aunque pueden advertirse vestigios de lo que hoy denominamos Psicología Jurídica mucho antes en el tiempo (vid. tabla 1), será en la década de los años ochenta del pasado siglo cuando se incorporen perfiles profesionales concretos dentro del sector, señalándose la década de los noventa como la edad de oro de la Psicología Jurídica española (Soria, 1998).

Sin embargo, la relación entre Psicología y Derecho no ha estado exenta de conflictividad debido a las peculiaridades de cada campo, superada a partir de la toma de conciencia jurídica de las ventajas de la colaboración (Lösel, 1992). En la actualidad esta relación está plenamente consolidada tornándose cada vez más fluida y con nuevos retos, atendiendo a la excesiva judicialización de las relaciones humanas (Garrido y Herrero, 2006).

Los tópicos de interés jurídico demandados a la Psicología han sido muchos y variados. Por otro lado, la propia Psicología ha delimitado objetos de estudio de interés propio dentro del Derecho. Esta confluencia de intereses ha dado lugar a distintas relaciones entre ambas ciencias (Bartol y Bartol,
1994; Muñoz, Bayés y Munné, 1980; Reichel, 1915) generando los distintos campos de intervención de los psicólogos jurídicos. Aunque han existido incursiones por parte de la Psicología en ámbitos más teóricos o filosóficos intentando explicar la esencia jurídica, las aportaciones de la Psicología al mundo del Derecho han sido eminentemente prácticas intentando dar respuestas a las demandas legales.

Este carácter aplicado de la Psicología Jurídica y su diversidad de campos de interés investigador y de actuación han provocado que en su conformación precisase del aporte de otros campos de la Psicología, especialmente la Psicología Social, Clínica y Experimental, viéndose su independencia comprometida a lo largo de la historia por intentos imperialistas de estos otros campos disciplinares, lo que ha dificultado la vertebración de la misma. A esta situación también se suma la falta de un itinerario formativo universitario independiente y consolidado. No será hasta la segunda mitad de los años noventa cuando este área comienza a estar presente en la Universidad (Catalán y González, 1987; Alfaro, Real, Tortosa, Jólluskin, 2004; Urra y Romero, 2006).

En la actualidad desde el punto de vista académico, la Psicología Jurídica cuenta en nuestro país con un corpus específico que ha dado lugar a la creación de publicaciones científicas especializadas (Anuario de Psicología Jurídica; Psicopatología Clínica, Legal y Forense; Colección Psicología y Ley; European Journal of Psychology Applied to Legal Context), a la edición de manuales específicos y a la inclusión de asignaturas concretas en los planes de estudio de las licenciaturas y post-grados en 
Tabla 1. Hitos en el desarrollo histórico de la Psicología Jurídica en España

- 1880: José $\mathrm{M}^{\mathrm{a}}$ Esquerdo defiende ante la Comisión de Reforma del Código Penal del Senado la falta de responsabilidad criminal del enajenado mental tras participar como perito en el caso de "El Sacamantecas".

- 1891: Se organiza el cuerpo de Facultativos Forenses en tres secciones: Medicina y Cirugía, Toxicología y Psicología, Medicina Mental y Antropología (Ley de Sanidad de 1885).

- 1898: Giner de los Ríos funda el Instituto Español Criminológico.

- 1903: Se funda la Escuela de Criminología en la Universidad Central de Madrid.

- 1903: Celebración en Madrid del congreso internacional sobre Medicina y Antropología Criminal.

- 1907-1936: La Junta de Ampliación de Estudios otorga becas que permiten la formación con algunos de los mejores especialistas europeos y americanos en Psicología Jurídica.

- 1918: Creación del Laboratorio de Criminología de la Facultad de Ciencias Jurídicas y Sociales de la Universidad Central .

- 1936-1939: Guerra Civil en España y exilio de las principales figuras de la Psicología Jurídica que crearán escuela en algunos países europeos y en Sudamérica .

- 1965: Creación del primer Gabinete Psicológico en la prisión de hombres de Madrid.

- Ley 38/1970 de 22 de diciembre: creación del Cuerpo Técnico de Instituciones Penitenciarias.

- 1971: El Colegio de Abogados de Barcelona crea la Sección de Psicología y Sociología Jurídicas.

- 1974: El Colegio de Abogados de Barcelona edita el Anuario de Sociología y Psicología Jurídica.

- 1975: El Colegio de Abogados de Barcelona crea el Instituto de Psicología Jurídica.

- 1976: Se organizan en Barcelona las I Jornadas Internacionales de Psicología Jurídica.

- 1983: Primeros psicólogos forenses de forma experimental en los Juzgados de Familia.

- 1985: Se elabora el Catálogo de Documentos Selectos para la formación y práctica en el ámbito de la Psicología Jurídica por encargo de la Junta Rectora de la Delegación de Madrid del C.O.P. .

- 1986: Se convoca el primer concurso oposición para cubrir plazas de psicólogos adscritos a la Administración de Justicia.

- 1987: Se crea la Sección de Psicología Jurídica del COP-M.

- 1989: Se crea la Sección de Psicología Jurídica del COP-C.

- 1990: Se crea la Sección de Psicología Jurídica del COP-España.

- 1990: Se celebra el II Congreso Nacional del COP con la inclusión específica del área de Psicología Jurídica.

- 1991: Se celebra el Congreso Hispano-Británico de Expertos en Psicología Jurídica en Pamplona.

- 1991: Se edita el Anuario de Psicología Jurídica del COP. Delegación de Madrid y Secretaria Estatal.

- 1993: Se crea el Master en Psicología Jurídica. U.N.E.D.-Fundación Universidad Empresa.

- 1993: Se crea la Asociación de Psicólogos, Educadores y Trabajadores Sociales de la Administración de Justicia.

- 1994: Se celebra en Barcelona la IV Conferencia Europea de Psicología y Ley.

- 1994: Se desarrolla en Madrid el I Encuentro sobre Mediación.

- 1995: Se crea la Sección de Análisis del Comportamiento Delictivo (S.A.C.D.) dentro de la Unidad Técnica de Policía Judicial de la Guardia Civil.

- Ley 35/1995 de ayudas y asistencia a las víctimas de delitos violentos y contra la libertad sexual. Promueve la creación de los Servicios de Atención a Víctimas (S.A.V.).

- 1997: Se crea el Curso de Experto en Psicología Jurídica del COP-M.

- 1997: Se constituye la Asociación de Técnicos de IIPP (ATIP).

- 1997: Se crea el Master en Psicología Clínica, Legal y Forense (UCM) con una unidad de peritación.

- 1998: El COP de España elabora el perfil profesional del psicólogo jurídico como especialidad específica de actuación del psicólogo.

- Ley Orgánica 5/2000 reguladora de la responsabilidad penal de los menores. Recoge la creación de un Cuerpo Nacional de Psicólogos Forenses.

- 2000: Se celebra el I Congreso Hispano-Alemán de Psicología Jurídica en Pamplona.

- 2000: Se constituye la Sociedad Española de Psicología Clínica, Legal y Forense. Publicación: Psicopatología Clínica, Legal y Forense.

- 2001: Se celebra en Madrid el IV Congreso de la Asociación Iberoamericana de Psicología Jurídica. .

- 2006: Se constituye la Sociedad Española de Psicología Jurídica y Forense. Publicación: Psicología y Ley.

- 2007: La Consejería de Justicia e Interior de la Comunidad de Madrid publica la "Guía Orientativa de Buenas Prácticas de Psicólogos Forenses". Elaborada por psicólogos forenses adscritos a la Administración de Justicia.

- 2009: Se edita la revista "The European Journal of Psychology Applied to Legal Context (Sociedad Española de Psicología Jurídica y Forense).

- 2010: Se crea el Instituto de Ciencias Forenses y de la Seguridad (ICFS) en la U.A.M. con amplia representación de la Psicología Jurídica.

- 2010: Se crea la Sección de Análisis de Conducta. Unidad Central de Inteligencia del Cuerpo Nacional de Policía.

Psicología, al menos hasta los nuevos itinerarios curriculares (Alfaro, Real, Tortosa y Jölluskin, 2004; Tortosa, Civera, Fariña y Alfaro, 2008).

En el plano aplicado, la Psicología Jurídica se sustenta por la existencia de distintos perfiles profesionales consolidados (e.g., psicólogos penitenciarios y psicólogos forenses) abriéndose camino en otros que las demandas sociales están impulsando, por ejemplo, psicología jurídica de la victimización criminal tras el protagonismo recuperado por la víctima dentro del modelo de política criminal actual; psicología criminalista ante los prometedores resultados que la aplicación de los métodos psicológicos están arrojando en su aplicación a la investigación del delito; o la psicología jurídica de la resolución de conflictos por el impulso de la justicia penal restaurativa y los esperanzadores resultados derivados de la aplicación de la mediación familiar. Única- 
mente es necesario un impulso en aras a la conceptualización y estructuración del campo para dejar de pensar en la Psicología Jurídica como un conjunto de meras aplicaciones de la Psicología al ámbito de la Ley (Clemente, 2010). En este sentido, los nuevos itinerarios curriculares elaborados a partir del Espacio Europeo de Educación Superior suponen una nueva amenaza al avance de la disciplina a favor de las especialidades aplicadas a la educación, la clínica y los recursos humanos.

\section{Hacia una definición consensuada}

El eclecticismo descrito en la conformación de la Psicología Jurídica y sus variados campos de investigación e intervención han contribuido a la utilización de conceptos restrictivos para su identificación definitorios de áreas específicas del campo global, apreciándose intentos hegemónicos de las áreas de mayor desarrollo sobre otras más incipientes. Nos encontramos así ante la utilización de distintos conceptos para abordar el campo de la Psicología y la Ley como Psicología Jurídica, Psicología Legal, Psicología Criminal, Psicología Forense, Psicología Judicial, etc. (Bajet, 1992).

Sin embargo, contar con un concepto unitario para dirigirse a un ámbito específico del saber es la primera condición para estructurar tanto el conocimiento teórico como aplicado del mismo, y por tanto dotarle de una entidad como disciplina científica.

Como se desprende de la lectura de las líneas anteriores, desde este artículo se propone el concepto de Psicología Jurídica para delimitar el campo de intersección entre la Psicología y el Derecho, siguiendo tanto nuestra tradición (Garrido, Masip y Herrero, 2006; Mira 1932; Muñoz, Bayés y Munné, 1980; Sobral, Arce y Prieto, 1994) como la de algunos países de nuestro entorno.

Podríamos definir la Psicología Jurídica como el ámbito de la Psicología que desarrolla sus investigaciones y metodología para mejorar el ejercicio del Derecho, en general, y la intervención del Sistema de Justicia en particular, entendiéndose por éste Fuerzas y Cuerpos de Seguridad, Administración de Justicia e Instituciones Penitenciarias.

\section{Delimitación de campos de investigación e intervención}

La amplia demanda jurídica y la propia iniciativa psicológica ha dibujado un amplio panorama de campos de interés tanto investigador como de intervención para los psicólogos jurídicos. Se entiende que en la mayoría de las ocasiones no se ha seguido un criterio técnico para aunar los mismos, provocando una agenda temática ingente y arbitraria. Desde este trabajo se propone la agrupación de los distintos ámbitos que conforman la Psicología Jurídica siguiendo dos criterios: uno académico-investigador y otro aplicado, claramente interrelacionados. Se proponen así nueve áreas dentro de la Psicología Jurídica:

1. Psicología Forense, pericial o psicología aplicada a los Tribunales. Rama de la Psicología Jurídica que desarrolla sus conocimientos y aplicaciones con vistas a concluir sus hallazgos en el seno de una Sala de Justicia con la finalidad de auxiliar al juzgador en la toma de decisiones (Soria, 2006). La función principal del psicólogo forense, con independencia del órgano judicial que solicite su intervención, será la emisión de informes periciales (medio de prueba). El psicólogo forense puede intervenir en el proceso judicial bien como perito designado judicialmente (psicólogos adscritos a la Administración de Justicia o designados por sorteo y lista corrida -art. 341.1 LEC-) o a instancia de parte.

Si bien, otros psicólogos especialistas en otras áreas de la psicología aplicada (clínica, neuropsicología, educativa, etc.) pueden actuar en la sala de justicia como peritos o expertos a requerimiento de los distintos operadores jurídicos (jueces, fiscales o abogados), será el psicólogo forense, por su formación específica, el psicólogo especialista en la realización de valoraciones psico-legales (puesta en relación de aspectos psicológicos con cuestiones jurídicas).

En la actualidad el psicólogo forense en nuestro país actúa en las siguientes jurisdicciones, además de la militar y canónica (vid. Tabla 2).

Este carácter aplicado de la Psicología Forense ha llevado a considerar este ámbito de la Psicología 
Jurídica como la única parte aplicada de la misma, utilizándose el concepto <forense $>$ para definir de forma global al campo, siguiendo la tradición de los países anglosajones (Esbec y Gómez-Jarabo, 2000). Como se desprende del presente artículo esta con-

Tabla 2. Actuación del psicólogo forense en las distintas jurisdicciones
ORGANO JURISDICCIONAL

JUZGADOS DE FAMILIA o PRIMERA INSTANCIA del Trabajo y de las Organizaciones aplicada al Sistema de Justicia).

Podría decirse que la Psicología Forense ha sido el sector de la Psicología Jurídica que mayor crecimiento ha experimentado en los últimos años tanto

\begin{tabular}{|c|c|}
\hline ORGANO JURISDICCIONAL & INTERVENCIÓN \\
\hline JUZGADOS DE FAMILIA o PRIMERA INSTANCIA & $\begin{array}{l}\text { - Procesos de guarda y custodia } \\
\text { - Procesos relacionados con el desarrollo del régimen de visitas } \\
\text { - Procesos de impugnación de tutelas } \\
\text { - Procesos de acogimiento familiar } \\
\text { - Procesos sobre adopción } \\
\text { - Privación de la patria potestad } \\
\text { - Nulidad civil del matrimonio } \\
\text { - Procesos de emancipación de menores de edad }\end{array}$ \\
\hline JUZGADOS DE INCAPACIDADES o PRIMERA INSTANCIA & $\begin{array}{l}\text { - Procesos de incapacitación } \\
\text { - Declaración de prodigalidad } \\
\text { - Idoneidad de tutor } \\
\text { - Proceso de tutela }\end{array}$ \\
\hline JUZGADOS DE LO PENAL o DE INSTRUCCIÓN & $\begin{array}{l}\text { - Valoración pericial psicológica del denunciado: capacidad procesal, imputabi- } \\
\text { lidad y riesgo delictivo } \\
\text { - Valoración pericial psicológica de la víctima: lesión y/o secuela psíquica, cre- } \\
\text { dibilidad del testimonio en abuso sexual infantil }\end{array}$ \\
\hline JUZGADOS DE VIGILANCIA PENITENCIARIA & $\begin{array}{l}\text { - Resolución de recursos referentes a la clasificación inicial y a progresiones y } \\
\text { regresiones de grado } \\
\text { - Resolución de recursos sobre la puesta en libertad condicional de los penados } \\
\text { y acordar las revocaciones que procedan } \\
\text { - Autorizar los permisos de salida cuya duración sea superior a dos días, excep- } \\
\text { to los clasificados en tercer grado } \\
\text { - Seguimiento de las medidas de seguridad }\end{array}$ \\
\hline JUZGADOS DE VIOLENCIA SOBRE LA MUJER & $\begin{array}{l}\text { - En materia penal: valoración del denunciado y de la supuesta víctima. Se } \\
\text { puede solicitar también la valoración de los menores cuando se haya ejercido } \\
\text { violencia sobre ellos } \\
\text { - En materia civil: guarda y custodia, régimen de visitas, acogimiento familiar, } \\
\text { procesos de adopción, privación de patria potestad y nulidad civil del matri- } \\
\text { monio } \\
\text { - Actuación asistencial: intervención en crisis (juicios rápidos y órdenes de pro- } \\
\text { tección) }\end{array}$ \\
\hline FISCALÍAS Y JUZGADOS DE MENORES & $\begin{array}{l}\text { - Valoración de la situación psicosocial del menor infractor para orientar respec- } \\
\text { to a la medida socioeducativa más adecuada } \\
\text { - Conciliación y reparación } \\
\text { - Seguimiento de las medidas postsentencia } \\
\text { - Supervisión de permisos y cambios de medida }\end{array}$ \\
\hline
\end{tabular}

Elaboración propia a partir de la Guía Orientativa de Buenas Prácticas de Psicólogos Forenses, editada por la Consejería de Justicia e Interior de la Comunidad de Madrid.

ceptualización se ofrece excesivamente restrictiva al no abarcar a todos los ámbitos de la Psicología Jurídica que sin aportar sus conocimientos y técnicas a la sala de justicia para asesorar al juez o tribunal en su toma de decisiones tienen una clara vertiente práctica (e.g., Psicología Judicial, Psicología Jurídica de la Resolución de Conflictos o Psicología desde el ámbito público como privado (Vázquez, 2008). El gran reto de la Psicología Forense actual, desde un punto de vista técnico, versa sobre dos áreas: a) la creación de instrumentos y métodos de evaluación apropiados al contexto de exploración forense (que sean capaces de sortear la actitud defensiva y manipulativa de los peritados mante- 
niendo elevados parámetros de fiabilidad y validez) $\mathrm{y}$ al objeto de la misma (que permitan realizar inferencias psico-legales); y b) consolidar criterios técnicos basados en la evidencia empírica para apoyar sus consideraciones periciales. Junto con las aportaciones al campo de las repercusiones legales de los trastornos mentales, el psicólogo forense enriquece la exploración pericial de la esfera psíquica contribuyendo a la individualización de la resolución judicial al ofrecer la posibilidad de realizar un análisis global e integral de la realidad psicológica del ser humano (emociones, motivaciones, esquemas mentales, proceso socializador, procesos básicos, etc.) (Díez Ripollés, 1990).

En el caso de los psicólogos forenses adscritos a la Administración de justicia, a pesar de que dicha figura profesional entra en el organigrama de la Institución en el año 1983 de forma experimental y desde el año 1987 de manera consolidada, tanto desde el sector de la judicatura como desde el órgano colegial se lleva tiempo reclamando un estatuto orgánico propio para el psicólogo forense a semejanza del que disfruta el médico forense (Chacón, 2008; II Jornadas de Jueces y Magistrados de Familia e Incapacidades, citado en López, 2007), y la acreditación antes de la toma de posesión de su cargo de un formación especializada y propia para el ejercicio de sus funciones, situación que se paliaría con la creación de procesos selectivos específicos para este perfil profesional, un periodo formativo en la Escuela Judicial y otro de rotación por las distintas jurisdicciones. Se completaría esta propuesta con la creación de un Cuerpo Nacional de Psicólogos Forenses como preveía la Ley Orgánica 5/2000 reguladora de la responsabilidad penal de los menores.

Para los psicólogos forenses que desarrollan su actividad desde el ámbito privado o los que pertenecen a la lista de peritos judiciales, el Colegio Oficial de Psicólogos a través de sus distintas delegaciones está trabajando en la elaboración de acreditaciones profesionales que regulen los criterios técnicos necesarios para contribuir a una adecuada praxis.

2. Psicología Penitenciaria. A partir de la Ley $38 / 70$ de 22 de diciembre el psicólogo forma parte del personal técnico de Instituciones Penitenciarias como un claro campo de aplica- ción de la investigación proveniente de la Psicología Criminal o Psicología de la Delincuencia (vid. infra). El psicólogo penitenciario a diferencia del psicólogo forense tiene un estatuto orgánico propio con funciones claramente delimitadas en la Ley Orgánica General Penitenciaria (L.G.P. 1/79) y en el Reglamento Penitenciario (Real Decreto 1201/81). Las actuaciones del psicólogo penitenciario pueden dividirse, fundamentalmente, en dos amplias áreas:

a) Evaluación de los internos y participación en los equipos técnicos y juntas de tratamiento. Estos órganos se reúnen semanalmente para decidir, entre otras cosas, los siguientes aspectos: programa de tratamiento de los internos, posibilidad de permisos de salida, clasificación inicial, revisiones de grado, propuesta de libertad condicional, propuesta de beneficios penitenciarios, etc.

b) Por otro lado, el psicólogo penitenciario tiene entre sus funciones la realización de programas específicos de intervención con todo tipo de penados, tanto con los que están cumpliendo una medida privativa de libertad (régimen cerrado, régimen ordinario, régimen abierto y liberados condicionales) como con los que están cumpliendo una medida penal alternativa al ingreso en prisión (suspensiones y sustituciones de condena, trabajos en beneficio de la comunidad, etc.).

3. Psicología Criminalista o psicología jurídica aplicada a la función policial. Incluiría la aplicación de los conocimientos y métodos de la Psicología a la operativa de la investigación de delitos y faltas. Abarcaría los siguientes aspectos: a) aportaciones de la psicología jurídica a la investigación criminal (técnicas de entrevista aplicadas al interrogatorio policial, especialmente en casos de detenidos, supuestas víctimas o testigos especialmente vulnerables, discapacitados o con deterioro o trastorno mental, principalmente en apoyo de los agentes que prestan sus servicios en Unidades Especializadas como los S.A.F., S.A.M. 
GRUMEs y EMUMEs; perfilamiento aplicado a la captura de agresores sistemáticos; y autopsia psicológica en casos de muerte y/o desapariciones de etiología dudosa); b) aplicación de la psicología a la negociación en situaciones críticas (toma de rehenes, secuestros, atrincheramientos, y tentativas de suicidio); c) trabajos muy específicos con testigos protegidos, apoyo a agentes encubiertos y gestión de colaboradores e informadores. En la actualidad dentro de nuestras fronteras contamos con dos secciones policiales que abordarían este ámbito: una en la Guardia Civil de más larga creación (Sección de Análisis del Comportamiento Delictivo, desde 1995, en el Departamento de Análisis Criminal de la Unidad Técnica de Policía Judicial) y otra en el Cuerpo Nacional de Policía (Sección de Análisis de Conducta, desde 2010, en la Unidad Central de Inteligencia Criminal). Es importante terminar destacando que la Psicología Criminalista es un área incipiente dentro de la Psicología Jurídica española, y que debe ser diferenciada de las aportaciones tradicionales de la Psicología a otros ámbitos del mundo policial y que tradicionalmente se ha denominado Psicología Policial: que comprende las actitudes de selección para ingreso en los Cuerpo policiales o posterior especialización, la asistencia clínica a sus componentes y las tareas en los Centros de Formación (Ibáñez y González, 2002).

4. Psicología Jurídica aplicada a la resolución de conflictos. Es el ámbito de la Psicología Jurídica dirigida a investigar, desarrollar y aplicar métodos y técnicas psicológicas que faciliten la búsqueda de soluciones pacíficas y consensuadas al conflicto, mediante un proceso de mediación. Parece oportuno diferenciar la mediación, en tanto que técnica de resolución de conflictos, de otros métodos encaminados a tal objetivo, como son la negociación, la conciliación y el arbitraje. La mediación es la intervención en un conflicto de una tercera parte neutral e imparcial: el mediador, que orienta y guía a las partes en conflicto, mediante la utilización de diversas técnicas, para que puedan manejar y/o resolver su disputa y llegar a un acuerdo consensuado con el fin de resolver su conflicto, sin forzar ni imponer la resolución, propiciando el diálogo, resolviendo el problema y desarrollando soluciones alternativas.

En España, esta técnica comenzó a aplicarse en el ámbito de los conflictos familiares, hace ya más de una década (Bernal, 2006). Debido al interés suscitado y el éxito en su intervención además de introducirse de forma experimental dentro de la propia Administración de Justicia (mediación intrajudicial), la mediación se ha ido extendiendo a otros campos del Derecho: penal, civil, laboral y contenciosoadministrativa. También está introduciéndose en el contexto penitenciario para resolución de los conflictos surgidos de la convivencia en prisión (Ríos, 2005). En el contexto penal, la mediación es compatible con el procedimiento judicial. Pretende potenciar la reparación del daño causado a la víctima (además de la compensación civil por daños y perjuicios), y de evitar o disminuir la reacción penal, añadiendo así una nueva forma de encontrar soluciones para ambas partes (Fons, 2005). Especial consideración legal tiene la mediación en el proceso penal de menores (Ley 5/2000).

La esencia de la mediación está en los principios que se mantienen como estructura inalterable sobre la que construir proceso y acuerdos. Existe un amplio consenso tanto en los documentos internacionales (Recomendación del Comité de Ministros del Consejo de Europa, Libro Verde sobre modalidades alternativas de solución de conflictos en ámbito del Derecho civil y mercantil, Código de Conducta Europeo para los Mediadores, Propuesta de Directiva al Parlamento Europeo y al Consejo, sobre determinados aspectos de la mediación en materias civiles y comerciales, etc.) como en la doctrina, refrendado en la práctica de Asociaciones y en sus propuestas de código deontológico, en torno a cuáles han de ser los puntos cardinales de la mediación: voluntariedad de las partes, imparcialidad, neutralidad, confidencialidad y profesionali- 
dad, junto a la buena fe, la flexibilidad del proceso, el carácter personalísimo del mismo o el interés de ambas partes, lo que determinan el marco en el que se configura y ha de desarrollarse la mediación.

5. Psicología del Testimonio. La prueba testifical en sus diferentes expresiones (descripciones e identificaciones) es uno de los pilares probatorios en los distintos procesos judiciales, resultando de especial importancia en los procesos penales. La investigación en Psicología Cognitiva de los procesos básicos (atención, percepción y memoria, principalmente) resulta de enorme interés para la obtención y valoración de la prueba testifical. Los tópicos sobre los que ha versado mayoritariamente la investigación dentro de la Psicología del Testimonio han sido cuatro (Manzanero, 2008): a) los factores de influencia en la exactitud de los testimonios, ya que los testimonios pueden ser imprecisos atendiendo a distintos factores que pueden incidir en el proceso de codificación, almacenamiento y recuperación del recuerdo; b) los procedimientos de obtención de declaraciones que permitan obtener la mayor cantidad posible de información exacta, sin influencias negativas; c) la evaluación de la credibilidad del testimonio, ya que constituye una demanda histórica por parte del Derecho a la Psicología la elaboración de métodos de detección de testimonios falsos (por engaño o error); y d) las pruebas de identificación realizadas por testigos y la evaluación de su exactitud, que ayudarían a evitar, o al menos minimizar, algunos de los errores que se cometen y a valorar adecuadamente la actuación de los testigos (Manzanero, 2008). Así, la investigación en Psicología del Testimonio tiene dos claras aplicaciones profesionales que entroncan con algunas de las especialidades descritas anteriormente: por un lado, para el psicólogo criminalista (aplicaciones de técnicas de entrevista para el interrogatorio policial, elaboración de retratos robots o reconocimiento de sospechosos) y por otro, para el psicólogo forense (facilitar la toma de declaraciones de las partes y asesorar a los tri- bunales para una mejor valoración de la prueba testifical). Por último, la Psicología de los Procesos Cognitivos (y fundamentalmente la Psicología de la Percepción) tiene un papel importante en otras áreas del Derecho, como la que implica la elaboración de informes periciales en las causas referidas al plagio de patentes, marcas y propiedad intelectual (Manzanero, 2008).

6. Psicología Judicial. Comprendería la aplicación de la investigación en Psicología Social (cognitiva y de los grupos) al ámbito jurídico. Su interés investigador ha versado tradicionalmente en relación a dos grandes áreas: a) los fundamentos del comportamiento normativo (conducencia/inconducencia de la norma); la interpretación de la fundamentación psicológica y social de las leyes y del Derecho como una forma de ordenar y crear equilibrio y orden social tendría una clara aplicación en la teoría legislativa (contribución de la Psicología Jurídica a la elaboración de las Leyes) (Oceja y Fernández-Dols, 2006); y b) el análisis de los factores extrajurídicos que influyen en la toma de decisiones judiciales tanto en el caso de jueces y tribunales como de jurados (Fariña, Arce y Vila, 1999; De Paúl, 1995; Novo y Arce, 2003; Sobral, Arce y Prieto, 1994).

7. Psicología de la Victimización Criminal. Se encargaría del estudio de los efectos provocados por la vivencia de un proceso de victimización criminal (Baca, Echeburúa y Tamarit, 2006; Soria, 2006). La Psicología de la Victimización criminal abarcaría los siguientes aspectos: a) intervención psicológica con víctimas (intervención en crisis y tratamiento de psicopatología traumática); b) prevención de la victimización mediante el control de los factores de riesgo victimal (prevención inespecífica mediante planes integrales de prevención y prevención específica dirigida a población de riesgo) y c) prevención de la victimización secundaria, mediante la sensibilización de los profesionales al servicio del Sistema de Justicia y el acompañamiento de la víctima a lo largo del proceso judicial. 
8. Psicología Criminal o Psicología de la Delincuencia. Rama de la Psicología Jurídica que desarrolla investigaciones y genera conocimientos específicos en relación a la explicación de la conducta criminal. Sus tópicos de interés investigador versan sobre: a) explicación de la conducta delictiva; b) prevención y tratamiento; c) estudios de carreras delictivas; y d) predicción del riesgo de violencia (Garrido, 2005; Redondo y Andrés-Pueyo, 2007). Es clara también la aportación de este campo de la Psicología Jurídica a la Criminología. El modelo más predominante dentro de la Psicología Criminal actual es el de los factores de riesgo/factores de protección para cada tipología delictiva: menores infractores, maltratadores, agresores sexuales, y delincuentes violentos (Redondo, 2008). Dentro de este nuevo paradigma se generan teorías explicativas de la conducta criminal no desde la perspectiva etiológica sino desde el reconocimiento empírico de qué factores predictores se asocian y con qué fuerza o grado con determinadas conductas delictivas (Andrés-Pueyo, 2009). Se dispone así de listados de factores de riesgo y protección asociados a los distintos tipos de conducta delictiva, siendo algunos comunes a distintos tipos de criminalidad. Los distintos factores de riesgo tendrán un diferente efecto en relación a la expresión de conductas delictivas según el carácter general o específico para un tipo de criminalidad. Estos factores operan de modo interrelacionado, interdependiente $y$ en muchos casos simultáneo. La investigación en el ámbito de la predicción del riesgo debe centrarse en el análisis de la dinámica funcional interactiva que permita aclarar cuál es la contribución real de cada variable para cada tipo de conducta delictiva (Sobral, Romero, Luego y Marzoa, 2000). Dentro de este paradigma se ha acuñado el concepto de gestión del riesgo. La gestión del riesgo hace referencia a la aplicación de los conocimientos disponibles generados en los estudios de valoración del riesgo para minimizar la frecuencia actual de las conductas violentas y delictivas así como sus efectos y es un campo donde los expertos deben desarrollar nuevas estrategias de intervención para reducir la criminalidad (AndrésPueyo y Redondo, 2007). En el aspecto práctico, este campo de la Psicología Jurídica aporta sus conocimientos al psicólogo penitenciario fundamentalmente, al forense (valoración del riesgo de violencia, individualización de la pena o asesoramiento dentro del proceso penal de menores) y al psicólogo criminalista (perfiles criminales y tipologías delictivas).

9. Psicología del Trabajo y de las Organizaciones aplicada al Sistema de Justicia. Estaría orientada a la planificación de la organización y mejora del Sistema de Justicia (Fuerzas y Cuerpos de Seguridad, Administración de Justicia e Instituciones Penitenciarias). Abarcaría todas las funciones desarrolladas por la Psicología del Trabajo y de las Organizaciones: selección, evaluación y orientación de personal, formación y desarrollo del personal, marketing y comportamiento de los usuarios del Sistema de Justicia, condiciones de trabajo y salud, organización y desarrollo de recursos humanos, y asesoramiento a los puestos directivos. Dentro de nuestras fronteras el sector donde más se ha desarrollado este ámbito de la Psicología Jurídica ha sido el de las Fuerzas y Cuerpos de Seguridad con la denominada Psicología Policial (Soria, 2006; Clemente, Parrilla, Vidal, 1998).

\section{Propuesta de un Master Oficial con directrices propias en Psicología Jurídica}

La complejidad y especificidad del trabajo del psicólogo en el ámbito jurídico, con independencia de su campo de desempeño profesional, requiere una especialización y formación en consonancia con las pretensiones derivadas de la creación del Espacio Europeo de Educación Superior (Declaración de Bolonia, 1999): dotar al alumnado de los conocimientos y habilidades necesarias para el desempeño de perfiles profesionales concretos (Real Decreto 1393/ 2007)(Romero, 2009; Muñoz y González, 2008). 
La idiosincrasia del perfil profesional de los psicólogos jurídicos precisa de la elaboración de un Master con directrices propias en Psicología Jurídica, siendo un riesgo que su formación se diluya dentro de programas formativos en otras áreas (e.g., Master de Psicología Clínica y de la Salud). La Psicología Jurídica es un campo de especialidad profesional del psicólogo con una identidad definida y reconocida (COP, 1998), que no debe confundirse ni solaparse por otras. En la actualidad cuenta con investigación diferenciada, publicaciones especializadas, perfiles profesionales consolidados y otros en vías de expansión y amplia oferta formativa aunque no homogeneizada. En definitiva, únicamente necesita consenso para organizar en un tronco común toda la ingente investigación y práctica profesional surgida a lo largo de su desarrollo.

Una vez definida la Psicología Jurídica y establecidos sus campos de investigación y actuación resulta más fácil elaborar un programa formativo para todos aquellos profesionales que deseen enfocar su actividad profesional en este campo. Se plantea desde el presente artículo la siguiente propuesta como punto de partida para el debate:
Aplicada. El esfuerzo y rigurosidad de los profesionales pioneros de este ámbito ha contribuido al reconocimiento y prestigio social que hoy disfruta esta especialidad y al incremento de la demanda de profesionales en este campo.

Si bien, en el plano académico e institucional adolece de un reconocimiento oficial que puede explicarse por la dificultad para vertebrar un campo disciplinar con tan diversos ámbitos de intervención y tan ecléctico en su conformación.

Con el presente trabajo queremos llamar a la reflexión de académicos y profesionales de la Psicología Jurídica para organizar todo el trabajo realizado hasta la actualidad lo que facilitaría la consolidación de esta disciplina de la Psicología Aplicada. Una actitud pasiva ante el olvido que este campo ha sufrido en los nuevos itinerarios curriculares elaborados a partir del Espacio Europeo de Educación Superior o acatar intentos de subyugación por parte de otros campos disciplinares, sería un riesgo elevado para la identidad de la Psicología Jurídica y para el servicio que ésta presta a la sociedad.

\section{PRIMER AÑO}

- Psicopatología Criminal y Forense I: Criminodinamia y repercusiones legales de los trastornos mentales

- Psicopatología Criminal y Forense II: Procesos de victimización específicos y su impacto psicológico (psicopatología traumática)

- Psicología de la Delincuencia

- Psicología del Testimonio

- Aplicación de la Psicología a la Investigación del delito: Psicología Criminalista

- Psicología Judicial

- Métodos de Investigación en Psicología Jurídica

\section{SEGUNDO AÑO}

- Psicología Forense: El proceso de evaluación pericial psicológica. Elaboración y ratificación de informes periciales en las distintas jurisdicciones

- Psicología Penitenciaria: evaluación y tratamiento de personas condenadas penalmente

- Psicología de la Victimización Criminal: evaluación e intervención psicológica con víctimas de delitos violentos

- Psicología Jurídica aplicada a la Resolución de Conflictos: La Mediación

PERIODO DE PRÁCTICAS SUPERVISADAS*

TRABAJO FIN DE MASTER * El alumno elegirá un periodo de prácticas supervisadas a lo largo de un año lectivo en una de las siguientes áreas: Psicología Forense, Psicología Penitenciaria, Psicología de la Victimización
Criminal y Psicología de la Resolución de Conflictos.

\section{Conclusiones}

La Psicología Jurídica en España ha tenido un desarrollo vertiginoso dentro de la Psicología

\section{Referencias}

Alfaro, E., Real, S., Tortosa, F. y Jölluskin, G. (2004): El desarrollo académico de la psicolo- 
gía jurídica. Anuario de Psicología Jurídica, 99114.

Andrés-Pueyo, A. (2009). La predicción de la violencia contra la pareja. En E. Echeburúa, J. Fernández-Montalvo y P. del Corral: Predicción del riesgo de homicidio y de violencia grave en la relación de pareja. Valencia: Centro Reina Sofía. Serie Documentos $\mathrm{n}^{\mathrm{o}} 15$.

Andrés-Pueyo, A. y Redondo, S. (2007). Predicción de la violencia: entre la peligrosidad y la valoración del riesgo de violencia. Papeles del Psicólogo, 28, 147-156.

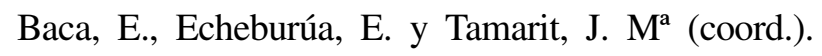
Manual de victimología. Valencia: Tirant lo Blanch.

Bajet, J. (1992). La Psicología Jurídica: pasado y presente de su breve historia. Anuario de Psicología Jurídica, 9-15.

Bartol, C. y Bartol, A. (1994). Psychology and law. Research and application. Pacific Grove: Brooks/ Cole Publishing Company.

Bernal, T. (2008). La mediación, una solución a los conflictos de ruptura de pareja. Madrid: Colex.

Carpintero, H. (2006). Breve historia de la psicología jurídica. En E. Garrido, J. Masip, y M. C. Herrero: Psicología Jurídica (pp. 43-75). Madrid: Pearson Educación.

Catalán, Mª J. y González, J. D. (1987). La necesidad de incluir la Psicología Jurídica dentro de los planes de estudio de Psicología. Papeles del Psicólogo, 30.

Chacón, F. (2008). Psicología Jurídica. Editorial. Guía del psicólogo, 281. Colegio Oficial de Psicólogos de Madrid.

Clemente, M. (2010). Psicología Jurídica. Una ciencia emergente explicativa del derecho. Madrid: Pirámide.

Clemente, M., Parrilla, A. y Vidal, M. A. (1998). Psicología jurídica y seguridad: policía y fuerzas armadas, Vol. I y II. Colección Retos Jurídicos en las Ciencias Sociales. Madrid: Fundación Universidad-Empresa.

Colegio Oficial del Psicólogo (1989). Perfiles profesionales. Recuperado de: http://www.cop.es/perfiles/.

Consejería de Justicia e Interior de la Comunidad de Madrid (2007). Guía Orientativa de Buenas
Prácticas de Psicólogos Forenses. Comunidad de Madrid.

De Paúl, P. (1995). El tribunal del jurado desde la psicología social. Madrid: Siglo XXI.

Díez Ripollés, J. L. (1990). Los elementos subjetivos del delito. Bases metodológicas. Valencia: Tirant lo Blanch.

Esbec, E. y Gómez-Jarabo (2000). Psicología forense y tratamiento jurídico-legal de la discapacidad. Madrid: Edisofer.

Fariña, F., Arce, R. y Vila, C. (1999). Efectos de la composición del jurado en los estilos deliberativos, análisis cognitivo y de contenido en la liberación. Anuario de Psicología Jurídica, 105-122.

Fons, G. (2005). La mediación en psicología jurídica. En M.A. Soria: Manual de psicología jurídica e investigación criminal (pp.229-248). Madrid: Pirámide.

Garrido, E. y Herrero, C. (2006). Relaciones entre la psicología y la ley (pp.3-42). En E. Garrido, J. Masip, y Ma . C. Herrero: Psicología Jurídica (pp. 43-75). Madrid: Pearson Educación.

Garrido, E., Masip, J. y Herrero, Mª.C.(2006). Psicología Jurídica. Madrid: Pearson Educación.

Garrido, V. (2005). Qué es la psicología criminológica. Madrid: Biblioteca Nueva.

Ibañez, J. y González, J. L. (2002). La psicología en las fuerzas y cuerpos de seguridad. En J. Urra (comp.). Tratado de psicología forense (pp. 489505). Madrid: Siglo XXI.

López, J. J. (2007). Custodia compartida. Cuestiones procesales. En A. Mª Saravia y J. J. García (Dir.): La jurisdicción de familia. Especialización. Ejecución de resoluciones y custodia compartida (pp. 249-295). Estudios de Derecho Judicial, 147. Consejo General del Poder Judicial.

Lösel, F. (1992). Psychology and law: overtures, crescendos and reprises. En F. Lösel, D. Bender y T. Bleisener: Psychology and law: International perspective (pp. 3-21). Nueva York: Walter de Gruyter.

Manzanero, A. (2008). Psicología del testimonio. Una aplicación de los estudios sobre la memoria. Madrid: Pirámide.

Manzanero, A. (2010). Hitos de la historia de la Psicología del Testimonio en la escena internacional. Boletín de Psicología, 100, 89-104. 
Mira, E. (1932). Manual de psicología jurídica. Barcelona: Salvat.

Muñoz Sabaté, L., Bayés, R. y Munné, F. (1980). Introducción a la psicología jurídica. México: Trillas.

Muñoz, J. M. y González, L. (2008). El necesario reconocimiento de la Psicología Jurídica como especialidad formativa y profesional. IV Congreso de Psicología Jurídica y Forense. Murcia: Mayo.

Novo, M. y Arce, R. (2003). Jueces: formación de juicios y sentencias. Granada: Grupo Editorial Universitario.

Oceja, L. V. y Fernández-Dols, J. M. (2006). La conducencia de la norma jurídica. En E. Garrido, J. Masip, y M. C. Herrero: Psicología Jurídica (pp. 77-108). Madrid: Pearson Educación.

Redondo, S. (2008). Individuos, sociedades y oportunidades en la explicación y prevención del delito: Modelo de Triple Riesgo Delictivo (TRD). Revista Española de Investigación Criminológica, 6, Artículo 7.

Redondo, S. y Andrés-Pueyo, A. (2007). La Psicología de la delincuencia. Papeles del Psicólogo, Vol. 28, 147-156.

Reichel, H. (1915). Sobre la psicología forense. Revista de Legislación, 126 y 127, 40-137.

Ríos, J. (2005). Mediación penitenciaria: reducir violencias en el sistema penitenciario. Madrid: Colex.
Romero, J. (2009). Editorial. Anuario de Psicología Jurídica, 19

Sáiz, M. y Sáiz, D. (2006). Aproximación histórica al desarrollo de la Psicología en el marco jurídico español. En M.A. Soria y D. Sáiz (Coord.): Psicología Criminal (pp. 1-23). Madrid: Pearson Educación.

Sobral, J., Arce, R. y Prieto, A. (1994). Manual de Psicología Jurídica. Barcelona: Paidós.

Sobral, J., Romero, E., Luengo, A. y Marzoa, J. (2000). Personalidad y conducta antisocial: amplificadores individuales de los efectos contextuales. Psicothema, 12, 661-670.

Soria, M. A. (Coord.) (2006). Psicología jurídica un enfoque criminológico. Madrid: Delta Ediciones.

Soria, M. A. (2006). La Psicología policial. En M. A. Soria y D. Sáiz (Coord.): Psicología Criminal (pp.275-310). Madrid: Pearson Educación.

Tortosa, F., Civera, C., Fariña, F. y Alfaro, E. (2008). La primera singladura de la psicología jurídica en España. Colección Psicología y Ley, 6, 11-38.

Urra, J. y Romero, J. (2006). La psicología jurídica en España. Infocop, 26, 4-11.

Vázquez, B. (2008). Aportaciones de la Psicología a la modernización del Sistema de Justicia Español. Conferencia Inaugural del IV Congreso de Psicología Jurídica y Forense, Murcia 8,9 y 10 de mayo de 2008. Anuario de Psicología Jurídica, $18,5-10$.

Manuscrito recibido: 16/12/2010

Revisión recibida: 01/02/2011

Aceptado: 11/03/2011 